\title{
¿Qué hacer cuando hay oposición médica al farmacéutico? (Caso de reinicio con clozapina)
}

\author{
Adrián Bartoll Andrés', Juan Salvador Escoda Casanova', Emilio Pol Yanguas ${ }^{1,2}$ \\ 1. Universidad Miguel Hernández de Elche. Facultad de Farmacia. 2. Centro Asistencial Doctor Esquerdo, Alicante.
}

\section{PALABRAS CLAVE}

clozapina,

oposición,

psiquiatría,

dosis,

delirium

\section{KEYWORDS}

clozapine,

disagreement,

psychiatrist,

dose,

delirium

\section{RESUMEN}

A. B. es un paciente con esquizofrenia y tiene pautada clozapina. Tras una serie de problemas con su medicación, presenta un episodio de agresividad contra su psiquiatra y el enfermero. El médico le retira clozapina y tras un periodo sin tomarla decide reinstaurarla a las dosis que tenía pautadas antes del episodio agresivo. El farmacéutico se opone a esta medida, y apoyándose en la información de la ficha técnica, afirma que la reinstauración de clozapina debe ser desde la dosis inicial, y semanalmente hacer controles hematológicos. Tras discusiones entre médico y farmacéutico sobre este caso, el médico persiste en desoír las advertencias del farmacéutico. Finalmente, debido a la insistencia desde el servicio de farmacia, se consigue empezar la pauta de clozapina correctamente, evitando de esta manera, un posible problema relacionado con el medicamento.

What to do in the event of physician disagreement with the pharmacist? (Case of reintroduction of clozapine)

\section{ABSTRACT}

$\mathrm{AB}$ is a schizophrenic patient receiving clozapine. Following a series of problems with the medication, the patient had an episode of aggressiveness against his psychiatrist and nurse. The physician suspended clozapine, and after a period without the drug, he decided to reintroduce it at the doses administered before the episode of aggressiveness. The pharmacist was against this decision and, based on the information contained in the Summary of Product Characteristics, considered that the reintroduction of clozapine should be made from the starting dose, with weekly blood controls. Following discussions between the physician and pharmacist on this subject, the former insists on paying no attention to the warnings of the pharmacist. Finally, after insistence from the Department of Pharmacy, it proved possible to prescribe clozapine correctly, thereby avoiding a possible drug-related problem. 


\section{Antecedentes}

A. B. es un paciente que proviene de una familia desestructurada, tiene antecedentes familiares de drogodependencia y enfermedad mental. Se le diagnostica esquizofrenia hebefrénica. En 2005 es internado en una institución para el tratamiento de enfermedades mentales por presentar pseudoalucinaciones cenestésicas y auditivas, que le llevaban a presentar conductas que el entorno vivía como amenazantes. Se le pautó valproato y clozapina, obteniéndose cierta mejora, hasta que durante un permiso, abandonó el tratamiento y su situación empeoró.

Hasta el verano de 2013, el paciente ha tenido diversos episodios de heteroagresividad, teniendo también periodos largos de estabilidad.

\section{Exposición del caso}

El problema surge el mes de agosto de 2013, cuando presentó un estado alterado, violento y agresivo, llegando a golpear físicamente a su psiquiatra y al enfermero. Este hecho se produjo tras varias amenazas por parte del paciente al estar en desacuerdo con su tratamiento, puesto que, según él, deberían retirarle clozapina, y pautarle aripiprazol.

Este suceso de agresividad ocurrió el 16/08/2013 por la mañana, después le retiraron la medicación oral, incluida clozapina, puesto que le encamaron con sujeción mecánica, y se le administró por vía intramuscular tratamiento antipsicótico (haloperidol y levomepromazina). Clozapina no fue pautada de nuevo hasta el 20/08/2013. En la preparación del tratamiento para el día 20/08/2013 (que se realiza el día anterior por la tarde en el servicio de farmacia) (tabla 1), no aparece todavía clozapina. Fue puesta a dispensar ese día, pero ya para comenzar el día siguiente, por lo tanto, clozapina se retiró el 16 por la mañana, lo que al sumar los días sin medicación resultan 5 días).
Tabla 1 Medicación pautada el 20/08/2013

\begin{tabular}{|l|c|c|}
\hline Medicamento & Pauta & Total \\
\hline Atorvastatina $40 \mathrm{mg}$ & $1-0-0$ & $40 \mathrm{mg}$ \\
\hline Biperideno $4 \mathrm{mg}$ oral/5 mg IM & $1-0-0 / 1-1-1$ & $19 \mathrm{mg}$ \\
\hline Haloperidol IM $5 \mathrm{mg}$ & $1-1-1$ & $15 \mathrm{mg}$ \\
\hline Levomepromazina IM $25 \mathrm{mg}$ & $1-1-1$ & $75 \mathrm{mg}$ \\
\hline Omeprazol $20 \mathrm{mg}$ & $1-0-0$ & $20 \mathrm{mg}$ \\
\hline Valproato $500 \mathrm{mg}$ & $2-1-2$ & $2.500 \mathrm{mg}$ \\
\hline
\end{tabular}

Tras esta complicada situación, hubo un desacuerdo entre el psiquiatra y el farmacéutico, debido a mantener ambos distintos criterios a la hora de reanudar el tratamiento de este paciente. Desde el día 19/08/13, hubo discrepancias entre ambos, ya que el facultativo médico proponía instaurar la dosis de clozapina que tomaba el paciente en el momento de la suspensión, siendo esta de $500 \mathrm{mg}$.

\section{Evaluación}

El farmacéutico, defendía que tras una interrupción, si es mayor de 48 horas, la clozapina debe reinstaurarse, para evitar problemas relacionados con el medicamento, comenzando por una dosis de $25 \mathrm{mg}$ al día, y hacer controles hematológicos semanales previos a su elevación. Además concurrían factores de riesgos concomitantes como el uso de benzodiazepinas y neurolépticos depot (tabla 2).

Tabla 2 Caracteristicas de clozapina

\begin{tabular}{|c|c|c|c|}
\hline Situación & Dosis & Controles hematológicos & Dosis máxima \\
\hline Inicio & $\begin{array}{l}\text { - } 12,5 \mathrm{mg} ; 1 \text { o } 2 \text { veces el primer dia } \\
\text { - } 25 \mathrm{mg} ; 1 \text { o } 2 \text { veces el segundo día } \\
\text { - Si tolera bien, aumentar lentamente de } 25-50 \mathrm{mg} \\
\text { en } 2 / 3 \text { semanas. Hasta un rango de } 200-450 \mathrm{mg} / \\
\text { día }\end{array}$ & Semanal durante 18 semanas & \multirow{4}{*}{$\begin{array}{l}900 \mathrm{mg} \\
\text { A partir de los } 450 \mathrm{mg} \text { au- } \\
\text { menta el riesgo de efectos } \\
\text { adversos (en especial con- } \\
\text { vulsiones) }\end{array}$} \\
\hline Mantenimiento & $\begin{array}{l}\text { Dentro del rango, con la menor dosis efectiva, } \\
\text { durante un mínimo de } 6 \text { meses }\end{array}$ & Mensuales & \\
\hline Retirada & $\begin{array}{l}\text { Reducción gradual de la dosis durante } 1 \text { o } 2 \text { semanas, } \\
\text { si es necesaria la retiración brusca, observación } \\
\text { clínica para evitar reacciones por retirada }\end{array}$ & Al mes de retirarlo & \\
\hline $\begin{array}{l}\text { Reinicio del } \\
\text { tratamiento } \\
\text { (tras interrupción } \\
\text { mayor o igual a } \\
48 \text { horas) }\end{array}$ & $\begin{array}{l}\text { - } 12,5 \text { mg; } 1 \text { o } 2 \text { veces el primer día } \\
\text { - } 25 \text { mg; } 1 \text { o } 2 \text { veces el segundo día } \\
\text { - Si tolera bien, aumentar más rápidamente de 25- } \\
50 \mathrm{mg} \text { en } 2 / 3 \text { semanas. Hasta un rango de } 200 \text { - } \\
450 \mathrm{mg} / \mathrm{dia}\end{array}$ & $\begin{array}{l}\text { Más de } 3 \text { dias y menos de } \\
4 \text { semanas } \rightarrow \text { semanal durante } \\
6 \text { semanas } \\
\text { Si es más de } 4 \text { semanas } \\
\rightarrow \text { igual que a inicio de } \\
\text { tratamiento }\end{array}$ & \\
\hline
\end{tabular}


Tabla 2 (Continuación) Características de clozapina

\begin{tabular}{|c|c|c|}
\hline Interacciones farmacológicas & Efectos adversos & Precauciones \\
\hline Benzodiazepinas $\rightarrow$ aumento de riesgo de colapso circulatorio & \multirow{6}{*}{$\begin{array}{l}\text { Taquicardia, cambios en ECG, } \\
\text { trastornos sanguíneos y lin- } \\
\text { fáticos, somnolencia, mareos, } \\
\text { convulsiones (a dosis altas) } \\
\text { hipersalivación, estreñimien- } \\
\text { to, incontinencia urinaria, au- } \\
\text { mento de peso, hipotensión } \\
\text { postural, fatiga, fiebre, au- } \\
\text { mento de transaminasas }\end{array}$} & \multirow{6}{*}{$\begin{array}{l}\text { Si es una dosis de más de } \\
200 \text { mg, dando la fracción de } \\
\text { dosis mayor antes de dormir } \\
\text { Si no excede de } 200 \mathrm{mg} / \mathrm{dia} \text {, } \\
\text { dar una sola dosis antes de } \\
\text { dormir } \\
\text { Para el reinicio de tratamien- } \\
\text { to, especial atención si el } \\
\text { paciente ha experimentado } \\
\text { eventos cardiacos o un paro }\end{array}$} \\
\hline Anticolinérgicos $\rightarrow$ aumento riesgo de delirium & & \\
\hline Antihipertensivos $\rightarrow$ potencia el efecto hipotensor & & \\
\hline Alcohol, IMAO, depresores SNC $\rightarrow$ aumento efectosedante & & \\
\hline Litio $\rightarrow$ aumento de riesgo de sufrir SNM* & & \\
\hline $\begin{array}{l}\text { Inductores (omeprazol)/inhibidores (cafeina) del CYP1A2 } \rightarrow \text { Inductores } \\
\text { provocarán disminución de concentraciones de clozapina, inhibidores un } \\
\text { aumento de las concentraciones }\end{array}$ & & \\
\hline
\end{tabular}

* Sindrome neuroléptico maligno.

Clozapina es un antipsicótico atípico útil en el tratamiento de las esquizofrenias resistentes. Sin embargo, provoca una fuerte sedación, aumento de peso, tiene efecto hipotensor, se ha asociado a aparición de síndrome metabólico y casos de agranulocitosis. Actualmente, por sus efectos adversos, dentro de las guías de tratamiento de la psicosis, clozapina está propuesta como segunda elección, y a la hora de instaurar un tratamiento o reinstaurarlo, hay que seguir una pauta progresiva de aumento de la dosis (1).

El rechazo por parte del psiquiatra a la colaboración del farmacéutico que, apoyado en su responsabilidad profesional, hace propuestas sobre los tratamientos y reacciones adversas que se pueden presentar y que puede poner en peligro la salud del paciente, se enmarca dentro de las reservas que algunos facultativos médicos mantienen ante otros colectivos clínicos (2).

Al tratarse de un riesgo de esta magnitud, vemos un conflicto importante, en la cual, la solución debe ser el beneficio del paciente. Las indicaciones, posología, precauciones y reacciones adversas a los medicamentos, aparecen en la ficha técnica por haber sido información extraída de ensayos clínicos, teniendo éstos estudios más validez que la opinión de expertos y facultativos. En el artículo de Benckhuijsen et al. (3), se da a conocer un caso de reinicio de clozapina a las dosis habituales que utilizaba el paciente, tras un periodo de dos días sin tomarse la medicación, que desemboca en delirium. Nos encontramos, pues, ante el riesgo de que se produzca un resultado negativo de la medicación (RNM) de inseguridad como consecuencia de un problema relacionado con el medicamento (PRM) ocasionado por la prescripción de una dosis, pauta y/o duración inadecuada (4).

\section{Intervención}

La intervención del farmacéutico se orienta a que, apoyándose de la ficha técnica, la reinstauración de clozapina se realice a partir de la dosis inicial, realizando semanalmente los correspondientes controles hematológicos.

\section{Resultado}

Inicialmente el médico persiste en desoír las advertencias del farmacéutico, pero finalmente, debido a la insistencia desde el servicio de farmacia, se consigue empezar la pauta de clozapina correctamente, evitando de esta manera, un posible problema relacionado con el medicamento que podría haber provocado graves repercusiones en la salud del paciente.

\section{Referencias bibliográficas}

1. Agencia Española de Medicamentos y Productos Sanitarios. Ficha técnica de $\mathrm{Nemea}^{\odot}$ [Internet]. [Acceso 2/9/2013]. Disponible en: www.aemps.gob.es

2. De Almeida Neto AC, Chen TF. When pharmacotherapeutic recommendations may lead to the reverse effect on physician decision-making. Pharm World Sci. 2008;30(1):3-8. doi:10.1007/s11096-007-9143-X

3. Benckhuijsen JA, Keet IP. Delirium on re-starting clozapine after a short break in treatment. Tijdschr Psychiatr. 2007;49(9):661-5.

4. Grupo de Expertos. Foro de Atención Farmacéutica. Documento de Consenso. Madrid: Consejo General de Colegios Oficiales de Farmacéuticos; 2008. ISBN 978-84-6911243-4. 